\title{
Systematic Review: Recommendations for Rehabilitation in ASD and ID From Clinical Practice Guidelines
}

\author{
Jordan Wickstrom, PhD a , Kristin Dell'Armo, PhD ${ }^{\text {b, }}$ \\ Emma Salzman, PsyD c, Jessica L. Hooker, PhD ${ }^{d}$, \\ Abigail Delehanty, PhD ${ }^{\mathrm{e}}$, Somer Bishop, PhD ${ }^{\mathrm{c}}$, \\ Marc J. Tassé, PhD ${ }^{f}$, Amy M. Wetherby, PhD ${ }^{d}$, \\ Antonia M.H. Piergies, BA ${ }^{\mathrm{c}}$, Diane Damiano, PhD, PT ${ }^{\mathrm{a}}$, \\ Alexandra Rauch, PhD ${ }^{\mathrm{g}}$, Audrey Thurm, PhD ${ }^{\mathrm{h}}$
}

\footnotetext{
${ }^{a}$ Functional and Applied Biomechanics Laboratory, Clinical Center, National Institutes of Health, Bethesda, MD

b Center for Autism Spectrum Disorders, Nationwide Children's Hospital, Columbus, $\mathrm{OH}$

' Department of Psychiatry and Behavioral Sciences, Weill Institute for Neurosciences, University of California San Francisco, San Francisco, CA

${ }^{\mathrm{d}}$ Autism Institute, Florida State University College of Medicine, Tallahassee, FL

e Department of Speech-Language Pathology, Duquesne University, Pittsburgh, PA

${ }^{f}$ Nisonger Center, Ohio State University, Columbus, $\mathrm{OH}$

${ }^{g}$ Rehabilitation Programme, World Health Organization, Geneva, Switzerland

${ }^{\mathrm{h}}$ Neurodevelopmental and Behavioral Phenotyping Service, National Institutes of Mental Health (NIMH), National Institutes of Health, Bethesda, MD
}

\section{KEYWORDS}

Autism spectrum

disorder;

Guideline;

Intellectual disability;

Neurodevelopmental

disorders;

\begin{abstract}
Objective To identify and summarize clinical practice guidelines for autism spectrum disorder (ASD) and intellectual disability (ID) for the Package of Interventions for Rehabilitation for the World Health Organization (WHO).

Data Sources Academic databases, Google Scholar, guideline databases, and professional society websites were searched using the general criteria "ASD/ID" AND "rehabilitation" AND "guideline," restricted to English-only guidelines.
\end{abstract}

List of abbreviations: AGREE-II, Appraisal of Guidelines for Research and Evaluation II; ASD, autism spectrum disorder; ID, intellectual disability; WHO, World Health Organization.

Supported (in part) by the Intramural Research Program of the NIMH (1ZICMH002961).

Disclosures: Alexandra Rauch works for the World Health Organization (WHO) in Head Quarters, Geneva. She alone is responsible for the views expressed in this publication, and she does not necessarily represent the views, decisions, or policies of WHO. The other authors have nothing to disclose.

Cite this article as: Arch Rehabil Res Clin Transl. 2021;3:100140 
Neurobehavioral manifestations; Rehabilitation; Therapeutics
Study Selection Work group members independently screened titles and abstracts (1952 ASD; 1027 ID) and excluded articles if not (1) a guideline; (2) about rehabilitation; (3) published since 2008; or (4) about ASD/ID. Full-text screening (29 ASD; 5 ID) involved 3 additional exclusion criteria: (1) contained conflict of interest; (2) lacked information on strength of recommendation; or (3) failed the Appraisal of Guidelines for Research and Evaluation II instrument. Six guidelines (4 ASD: 2 on youth, 1 on adults, 1 on all ages; 2 ID: 1 on challenging behaviors, 1 on mental health) resulted.

Data Extraction Work group members extracted 524 recommendations (386 ASD; 138 ID) from the guidelines including the level of evidence, diagnostic and age group, recommendation type (assessment, intervention, service), target, and valence.

Data Synthesis Of the 270 intervention recommendations (212 ASD; 58 ID), only 36 for ASD and 47 for ID were empirically based. Most comprised biomedical (23\%), pharmacologic (29\%), and psychosocial (21\%) interventions for ASD and behavioral (14\%), pharmacologic (29\%), and psychological (14\%) interventions for ID. Intervention recommendations primarily targeted coexisting conditions (56\% ASD; 93\% ID), whereas core symptoms received much less attention (26\% ASD).

Conclusions Clinical practice guidelines reviewed for ASD and ID primarily contained recommendations based on expert opinion, with the plurality of recommendations relating to pharmacologic treatment. Vital next steps include identifying relevant interventions for inclusion in the WHO Package and continuing to conduct rigorous intervention research, particularly on core symptoms of these conditions, to extend recommendations for high-quality guidelines.

Published by Elsevier Inc. on behalf of American Congress of Rehabilitation Medicine. This is an open access article under the CC BY-NC-ND license (http://creativecommons.org/licenses/bync-nd/4.0/).
The World Health Organization (WHO) launched the Rehabilitation 2030 Call for Action initiative to call attention to and strengthen rehabilitation in health systems worldwide. ${ }^{1-3}$ Rehabilitation is among the primary services included in universal health coverage, so a Package of Interventions for Rehabilitation is being developed for national and subnational levels ${ }^{1}$ with a specific focus on low- and middle-income countries. The Package of Interventions for Rehabilitation will provide information on evidence-based interventions, how to perform such interventions, and the associated resource requirements that will help countries plan, budget, and integrate interventions across all service delivery platforms.

The development of the Package of Interventions for Rehabilitation follows a stepwise approach. ${ }^{1}$ Twenty health conditions were initially selected based on prevalence, associated level of disability (using disability weights), ${ }^{4}$ and expert opinion. The health conditions outlined for this package are congenital or acquired and noncommunicable in nature, wherein rehabilitation will improve or return an individual's functioning to its maximum. The selected health conditions do not present an exhaustive list because additional health conditions will be added later. ${ }^{1}$ As a first step in developing the package, technical working groups composed of clinical and research experts for each of the health conditions identified high-quality clinical practice guidelines and extracted relevant information. Clinical practice guidelines are derived from rigorous systematic reviews of the best available research and practice experience and involve high-quality methodology. They also primarily focus on the effectiveness of interventions, provide a framework for clinical decision making, and explain the benefits and harms of alternative care options predicated on evidence and value judgments.

Autism spectrum disorder (ASD) and intellectual disability (ID) are the selected health conditions discussed in this report. Both conditions are neurodevelopmental disorders in the fifth edition of the Diagnostic and Statistical Manual for Mental Disorders ${ }^{5}$ and the 11 th edition of the International Classification of Disease. ${ }^{6}$ ASD is a disorder with onset in the early developmental period that is characterized by deficits in social communication and the presence of restricted, repetitive patterns of behavior. ${ }^{5}$ ID is defined by deficits in both intellectual and adaptive functioning during the developmental period and is coded based on the severity of adaptive functioning deficits, which can range from mild to profound. ${ }^{5,6}$ By definition, individuals with ID in the mild range may live fairly independent lives, whereas individuals in the profound range typically require constant supervision. We combined the findings for ASD and ID in this report because these disorders often co-occur with similar aspects of adaptive functioning often affected. Although the exact prevalence of ASD in people with ID is difficult to determine, recent estimates indicate that ID is present in approximately $30 \%-40 \%$ of individuals with ASD. ${ }^{7,8}$ Many people with ASD or ID have associated comorbid conditions, such as challenging behavior, mental health concerns, or associated health issues, ${ }^{9,10}$ which are often the focus of intervention.

The developmental progression of specific skills is generally expected to occur on a slower trajectory for people with ID and ASD (specifically in social communication skills for ASD) than for individuals without these conditions. Although rehabilitative interventions in ASD and ID, in contrast to disorders with an onset later in life, may not have the goal of restoring individuals to a previous level of functioning, goals may involve improving skills to maximize adaptive functioning in various domains (eg, daily living skills, communication, socialization). Additionally, optimizing functioning may include minimizing maladaptive effects of restricted interests and repetitive behaviors for those with ASD and improving cognitive functioning in those with ID. 
The purpose of this report is to summarize the results of the search (including the extraction) and the analysis of rigorously vetted clinical practice guidelines on rehabilitation in ASD and ID conducted by the respective technical working groups. The aims are to (1) detail the search strategies used to identify relevant clinical practice guidelines; (2) describe the screening process for determining eligible guidelines to be included in the Package of Interventions for Rehabilitation; (3) review the final selection of guidelines and corresponding recommendations; (4) present the recommendations categorized by type and target for each diagnostic group as well as by level of evidence; and (5) discuss research-to-practice gaps for future guideline development.

\section{Methods}

High-quality clinical practice guidelines were sought based on a systematic search to identify relevant interventions for rehabilitation. These guidelines were selected based on specific criteria developed by the WHO Rehabilitation Programme.

\section{Search strategy}

First, an extensive literature search was performed by both technical working groups for ASD and ID in 6 academic databases, 1 online search engine, 10 guideline databases, and 7 professional rehabilitation society websites (table 1). The following general search criteria were used: "health condition (ie, ASD or ID)" AND "rehabilitation" AND "guideline" and were limited to the English language (see supplemental table S1 for full electronic search strategy of academic databases, available online only at http://www.archives-pmr.org/). In accordance with criteria determined by the WHO Rehabilitation Programme, the search was also restricted to 2008-2019 (search performed in January 2019) for all 20 technical working groups and their respective health conditions to find current guidelines for intervention.

\section{Identification of suitable guidelines}

\section{Title and abstract screening}

Next, 2 members of each of the technical working groups independently screened the titles and abstracts of the articles retrieved from the search process. Articles were excluded for the following reasons: (1) not a guideline; (2) not about rehabilitation; (3) older than 10 years; or (4) developed for health conditions other than ASD or ID. The working group members then compared their decisions and discussed any discrepancies with a third member to reach consensus. In cases in which consensus could not be reached, the technical working group consulted with the WHO Rehabilitation Programme methods expert. Once the guidelines for inclusion were agreed on, the full-text versions were screened.

\section{Full-text screening}

The same process for the title and abstract screening was applied during full-text screening, along with 3 additional exclusion criteria: (1) the presence of a conflict of interest (financial or nonfinancial); (2) the absence of information on the strength of the recommendation; or (3) not passing the
Appraisal of Guidelines for Research and Evaluation (AGREEII) ${ }^{11}$ instrument criteria, which was applied to all guidelines that passed the first 6 exclusion criteria (items 1-4 in title and abstract screening and 1-2 in full-text screening). Three members of each technical working group independently determined AGREE-II ratings to assess the quality of each clinical practice guideline. All items were scored by each member, but only 9 AGREE-II items $(4,7,8,10,12,13,15$, 22,23 ) were specifically used for the selection of guidelines as predetermined by the WHO Rehabilitation Programme. ${ }^{1}$ If the rating of any item differed by more than 2 points among the 3 members, the score was discussed to reach consensus. In cases in which consensus could not be reached, the groups consulted the WHO Rehabilitation Programme methods expert. Guidelines were excluded if the sum of the mean scores of the selected items was $<45$, or if the mean score of the 3 researchers on items $7,8,12$ or 22 was $<3$. These criteria were determined by the WHO experts as essential for the identification of high-quality clinical practice guidelines.

\section{Data extraction from selected guidelines}

Technical working groups extracted specific information outlined by the WHO Rehabilitation Programme. Because the goal of this report is to describe the current recommendations from vetted guidelines that exist for ASD and ID, only the recommendations from the guidelines and their corresponding level of evidence are reported here. For the purposes of this article, the reported level of evidence was categorized into 1 of 2 categories: "based on empirical evidence" or "based on expert opinion." The category "based on empirical evidence" consisted of varying levels of evidence, including randomized controlled trials, meta-analyses, systematic reviews, casecontrol or cohort studies, and nonanalytical studies (eg, case reports, case series). We combined all levels of evidence into this empirical evidence category because each guideline used its own grading system for prescribing level of evidence. Conversely, the category "based on expert opinion" (the term used in the guidelines) consisted of recommendations that arose from clinical consensus from members of the guideline development group in cases in which there was insufficient empirical data to prescribe a level of evidence. Additional classifiers were also included to describe the target focus of each recommendation: "diagnostic group" (ASD, ID), "general age group" (children, young people, adults), "type of service" (eg, assessment, intervention), "target of service" (what the recommendation was intended to help with), and "valence" (with a positive valence referring to "do recommend," a negative valence referring to "do not recommend," or a neutral valence referring to "insufficient evidence to recommend"). Recommendations could be classified into more than 1 category for both type and target of service. To identify these categories, 3 working group members reviewed each recommendation and agreed on categorizations through a 3step process: (1) the 3 members typed the specific word(s) used in the actual recommendation into the applicable table column; (2) the team members grouped similar words from step 1 into overarching categories; and (3) the team members confirmed accuracy and consistency of decision making for all categorizations applied in step 2. 
Table 1 Results from the literature search and screening process

\begin{tabular}{|c|c|c|c|}
\hline \multicolumn{4}{|c|}{ Search Results } \\
\hline \multicolumn{2}{|l|}{ Academic Databases } & ASD Group & ID Group \\
\hline \multicolumn{2}{|l|}{ 1. PubMed/MEDLINE } & 350 & 275 \\
\hline \multicolumn{2}{|l|}{ 2. Embase } & 540 & 261 \\
\hline \multicolumn{2}{|l|}{ 3. CINAHL Plus } & 197 & 220 \\
\hline \multicolumn{2}{|l|}{ 4. PEDro } & 1 & 0 \\
\hline \multicolumn{2}{|l|}{ 5. Scopus } & 1018 & - \\
\hline \multicolumn{2}{|l|}{ 6. Web of Science } & 618 & - \\
\hline \multirow{2}{*}{\multicolumn{4}{|c|}{ Search Engine }} \\
\hline & & & \\
\hline \multicolumn{2}{|l|}{ 1. Google Scholar (limited to 250, as suggested by the WHO) } & 250 & 250 \\
\hline \multirow{2}{*}{\multicolumn{4}{|c|}{ Guideline Databases }} \\
\hline & & & \\
\hline \multicolumn{2}{|l|}{ 1. Scotland's Guidelines International Network (G-I-N) } & 23 & 0 \\
\hline \multicolumn{2}{|c|}{ 2. United Kingdom's National Institute for Health and Care Excellence (NICE) } & 36 & 18 \\
\hline \multicolumn{2}{|c|}{ 3. Australia's National Health and Medical Research Council (NHMRC) } & 1 & 4 \\
\hline \multicolumn{2}{|l|}{ 4. Scottish Intercollegiate Guidelines Network (SIGN) } & 16 & 14 \\
\hline \multicolumn{2}{|c|}{ 5. Canadian Medical Association (CMA) Infobase: Clinical Practice Guidelines } & 0 & 1 \\
\hline \multicolumn{2}{|c|}{ 6. New Zealand Guidelines Group (NZGG) } & 7 & 75 \\
\hline \multicolumn{2}{|l|}{ 7. United States' National Guideline Clearinghouse (NGC) } & 47 & 34 \\
\hline \multicolumn{2}{|l|}{ 8. United Kingdom's eGuidelines } & 6 & 5 \\
\hline \multicolumn{2}{|c|}{ 9. United Kingdom's National Library for Health (NLH) Guidelines Database } & 190 & 87 \\
\hline \multicolumn{2}{|c|}{ 10. France's L'Agence Nationale d'Accréditation et d' Évaluation en Santé (ANAES) } & 3 & - \\
\hline & Subtotal & 329 & 238 \\
\hline \multicolumn{4}{|l|}{ Professional Rehabilitation Society Websites } \\
\hline \multicolumn{2}{|l|}{ 1. American Academy of Neurology (https://www.aan.com/) } & 0 & 0 \\
\hline \multicolumn{2}{|l|}{ 2. American Academy of Pediatrics (https: //www.aap.org/) } & 0 & 2 \\
\hline \multicolumn{2}{|c|}{ 3. American Academy of Child \& Adolescent Psychiatry (https://www.aacap.org/) } & 1 & 0 \\
\hline \multicolumn{2}{|l|}{ 4. American Psychiatric Association (https://www.psychiatry.org/) } & 0 & 0 \\
\hline \multicolumn{2}{|c|}{ 5. American Occupational Therapy Association (https://www.aota.org/) } & 1 & 0 \\
\hline 6. Society for Developmental \& Behavioral Pediatrics (http://w & & 0 & 0 \\
\hline 7. American Speech-Language-Hearing Association (https://wn & & 28 & 34 \\
\hline & Subtotal & 30 & 36 \\
\hline & Grand Total & 3333 & 1280 \\
\hline Screening Results & & & \\
\hline & ASD G & & ID Group \\
\hline Total after duplicate removal & 1952 & & 1027 \\
\hline Total after title and abstract screening & 29 & & 5 \\
\hline Total after full-text screening & 8 & & 4 \\
\hline Total after AGREE-II tool & 4 & & 2 \\
\hline
\end{tabular}

NOTE. - indicates the search was not performed.

Abbreviations: CINAHL, Cumulative Index to Nursing and Allied Health; PEDro, Physiotherapy Evidence Database.

\section{Results}

\section{Literature search and screening results}

A broad overview (fig 1) as well as a more detailed record (see table 1) characterize the process involved to obtain the resulting guidelines from the literature search for each group (ASD, ID).

\section{ASD guidelines}

The literature search resulted in a total of 3333 ASD articles across academic databases $(n=2724)$, search engines $(n=250)$, guideline databases $(n=329)$, and professional websites $(n=30)$. After duplicate articles were removed, a total of 1952 articles remained. Title and abstract screening reduced the number of articles to 29 guidelines, and fulltext screening reduced it to 8 guidelines. After applying the 


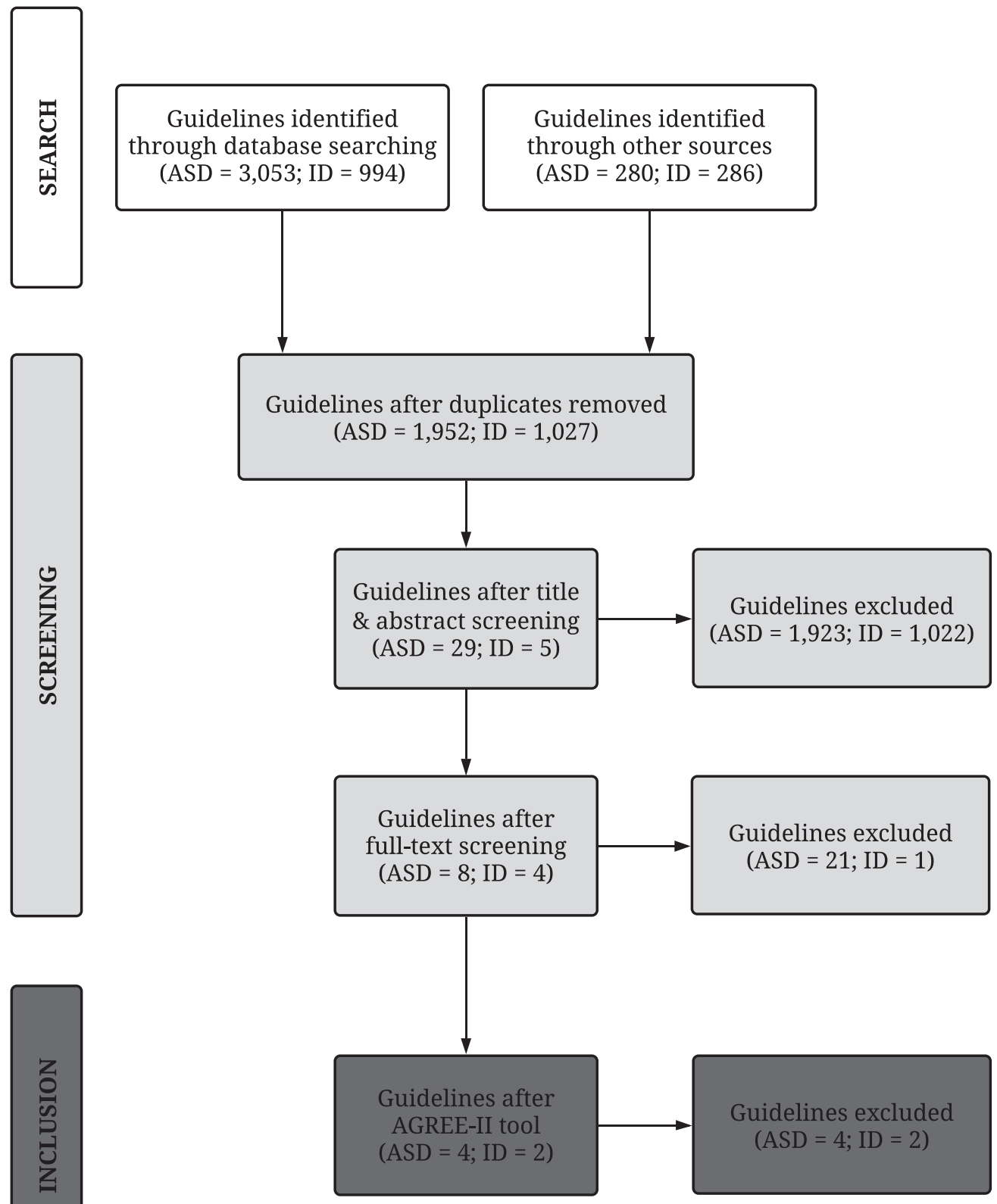

Fig 1 Flow chart of the results from the literature search and screening process.

AGREE-II instrument (table 2), 4 guidelines met the criteria, ${ }^{12-15}$ and their descriptions are provided (table 3 ). Two guidelines focused on treatment and support of youth, ${ }^{13,14} 1$ targeted diagnosis and treatment in adults, ${ }^{15}$ and 1 concentrated on assessment, diagnosis, and treatment of all ages. ${ }^{12}$

\section{ID guidelines}

A total of 1280 ID articles were found in the literature search across academic databases $(n=756)$, search engines $(n=250)$, guideline databases $(n=238)$, and professional websites $(n=36)$. After the removal of duplicate articles, 1027 articles remained. Title and abstract screening reduced the number of articles to 5 guidelines, and full-text screening to 4 guidelines. Two guidelines ${ }^{16,17}$ met the AGREE-II instrument criteria (see table 2) for data extraction and are described (see table 3 ). Both guidelines focused on prevention and treatment in individuals of all ages, with 1 guideline targeting challenging behaviors ${ }^{16}$ and the other mental health problems. ${ }^{17}$

\section{Data extraction results}

Data extraction results for level of evidence and the additional classifiers described in the Methods are provided in supplemental table S2 (available online only at http: / /www. archives-pmr.org/). For a visual breakdown of categories 
Table 2 Results from the AGREE-II instrument for the 6 included guidelines are provided, with numbers representing the average of 3 reviewers' scores. The 9 items highlighted in gray were used for the selection of guidelines as predetermined by the WHO Rehabilitation Programme, and guidelines were included if the sum of the mean scores of the selected items was at $\geq 45$ and if the mean scores on items $7,8,12$, and 22 were $\geq 3$

\begin{tabular}{|c|c|c|c|c|c|c|c|}
\hline AGREE-II Domain & AGREE-II Items & $\begin{array}{l}\text { SIGN } 145 \\
\text { (ASD) }\end{array}$ & $\begin{array}{l}\text { KCE } 233 \\
\text { (ASD) }\end{array}$ & $\begin{array}{l}\text { NICE CG170 } \\
(A S D)\end{array}$ & $\begin{array}{l}\text { NICE CG142 } \\
\text { (ASD) }\end{array}$ & $\begin{array}{l}\text { NICE NG11 } \\
\text { (ID) }\end{array}$ & $\begin{array}{l}\text { NICE NG54 } \\
\text { (ID) }\end{array}$ \\
\hline \multirow[t]{3}{*}{ 1-Scope and purpose } & Item 1 & 6 & 7 & 7 & 7 & 7 & 7 \\
\hline & Item 2 & 6 & 7 & 7 & 7 & 7 & 7 \\
\hline & Item 3 & 5 & 7 & 7 & 7 & 7 & 7 \\
\hline \multirow[t]{3}{*}{ 2-Stakeholder involvement } & Item 4 & 6 & 6 & 7 & 6 & 6 & 6 \\
\hline & Item 5 & 5 & 7 & 6 & 6 & 6 & 7 \\
\hline & Item 6 & 7 & 6 & 7 & 7 & 6 & 7 \\
\hline \multirow[t]{8}{*}{ 3-Rigor of development } & Item 7 & 6 & 7 & 7 & 7 & 7 & 7 \\
\hline & Item 8 & 3 & 6 & 6 & 6 & 6 & 7 \\
\hline & Item 9 & 5 & 7 & 7 & 6 & 7 & 7 \\
\hline & Item 10 & 2 & 7 & 5 & 5 & 5 & 6 \\
\hline & Item 11 & 7 & 7 & 7 & 7 & 7 & 7 \\
\hline & Item 12 & 5 & 6 & 7 & 7 & 7 & 7 \\
\hline & Item 13 & 6 & 7 & 6 & 6 & 6 & 4 \\
\hline & Item 14 & 6 & 4 & 5 & 7 & 7 & 7 \\
\hline \multirow[t]{3}{*}{ 4-Clarity and presentation } & Item 15 & 6 & 6 & 6 & 6 & 6 & 6 \\
\hline & Item 16 & 6 & 6 & 6 & 6 & 6 & 6 \\
\hline & Item 17 & 7 & 7 & 7 & 7 & 7 & 7 \\
\hline \multirow[t]{4}{*}{ 5-Applicability } & Item 18 & 2 & 5 & 2 & 1 & 2 & 2 \\
\hline & Item 19 & 6 & 4 & 6 & 3 & 5 & 4 \\
\hline & Item 20 & 4 & 2 & 7 & 6 & 7 & 6 \\
\hline & Item 21 & 4 & 1 & 4 & 3 & 1 & 1 \\
\hline \multirow[t]{3}{*}{ 6-Editorial independence } & Item 22 & 6 & 7 & 5 & 6 & 5 & 5 \\
\hline & Item 23 & 6 & 6 & 5 & 7 & 6 & 6 \\
\hline & Sum of highlighted items & 46 & 58 & 54 & 56 & 54 & 54 \\
\hline
\end{tabular}

Abbreviations: CG, Clinical Guideline; KCE, Belgian Healthcare Knowledge Centre; NG, NICE Guideline; NICE, National Institute for Health and Care Excellence; SIGN, Scottish Intercollegiate Guidelines Network.

arranged by type of service and target of service, refer to supplemental figs S1 and S2 (available online only at http: / / www.archives-pmr.org/), respectively. Of 524 (386 ASD; 138 ID) total recommendations, $52 \%$ focused specifically on intervention (212 ASD; 58 ID), which is the focus of the Package of Interventions for Rehabilitation and of this article. The percentage of intervention recommendations categorized by each type and target of intervention are presented in fig 2. For type of intervention, pharmacologic was the primary category, accounting for $29 \%$ of both ASD and ID intervention recommendations, followed by biomedical and psychosocial for ASD (23\% and 21\%, respectively) and behavioral and psychological for ID (14\% for each). For target of intervention, coexisting conditions were the primary category, encompassing $56 \%$ of ASD and $93 \%$ of ID intervention recommendations, whereas adaptive functioning was only $11 \%$ of ASD and $7 \%$ of ID intervention recommendations. Core symptoms were targeted in $26 \%$ of ASD recommendations. Regarding valence, only $67 \%$ of ASD compared with $100 \%$ of ID recommendations were categorized with a positive valence.

For both disorders, many of the intervention recommendations were based on expert opinion, with only $17 \%$ $(n=36)$ of ASD and $81 \%(n=47)$ of ID recommendations based on empirical evidence. The percentage of intervention recommendations based on empirical evidence are categorized by each type and target of intervention (fig 3). Of these evidence-based intervention recommendations, the same trends remained with pharmacologic as the primary type of intervention accounting for $36 \%$ of both ASD and ID intervention recommendations, followed by biomedical and psychosocial for ASD (19\% for each) and behavioral and psychological for ID (17\% for each). For target of intervention, coexisting conditions remained as the primary category with evidence-based intervention recommendations, accounting for 39\% of ASD and $100 \%$ of ID recommendations. Most of the ASD and ID recommendations $(61 \%$ ASD, $100 \%$ ID) had positive valence. Regarding age group, $12 \%$ were about children only $(n=4$ ASD, $n=6$ ID); $22 \%$ were about children and young people, $(n=18$ ASD, $n=0$ ID); $48 \%$ were about children, young people, and adults ( $n=0$ ASD, $n=40$ ID); and $18 \%$ were about adults only $(n=14$ ASD, $n=1$ ID).

\section{Discussion}

This report included an extensive review of the literature to identify and extract information from clinical practice guidelines for ASD and ID for potential inclusion in the WHO Rehabilitation Programme's Package of Interventions for Rehabilitation. Very few guidelines (6) regarding rehabilitation for ASD and ID met criteria for inclusion, all of which were produced by government agencies. Across the 6 
Table 3 Descriptions of each guideline consisting of the organization, guideline number, title, reference, year published, and scope

\begin{tabular}{|c|c|}
\hline Organization (Guideline No.) & Title \\
\hline $\begin{array}{l}\text { Scottish Intercollegiate } \\
\text { Guidelines Network } \\
\text { (SIGN 145) }\end{array}$ & $\begin{array}{l}\text { Assessment, diagnosis } \\
\text { and interventions for } \\
\text { autism spectrum } \\
\text { disorders: a national } \\
\text { clinical guideline }^{12}\end{array}$ \\
\hline $\begin{array}{l}\text { Belgian Health Care } \\
\text { Knowledge Centre } \\
\text { (KCE 233) }\end{array}$ & $\begin{array}{l}\text { Management of autism in } \\
\text { children and young } \\
\text { people: a good clinical } \\
\text { practice guideline }^{13}\end{array}$ \\
\hline
\end{tabular}

Year Scope (Text Taken From Each Guideline)

2016 This guideline provides recommendations based on current evidence for best practice in the assessment, diagnosis, and interventions for children, young people, adults, and older adults with ASD. It includes screening and surveillance, diagnosis, and assessment, clinical interventions, and service provision, as well as recommendations for research and audit.

2014 This guideline provides recommendations based on current scientific evidence for treatment and support of children and adolescents with autism and their family. The objective of treatment is to improve outcomes specific to a particular domain of ASD, thereby improving the overall outcome for the child or adolescent with ASD. Caregivers are encouraged to interpret these recommendations in the context of the individual situation and to take into account the values and preferences of the children and adolescents and of their families.

National Institute for Health and Care Excellence (NICE CG170)

National Institute for Health and Care Excellence (NICE CG142)

National Institute for Health and Care Excellence (NICE NG11)

National Institute for Health and Care Excellence (NICE NG54)
Autism spectrum disorder in under 19s: support and management ${ }^{14}$
2013 This guideline covers children and young people with autism spectrum disorder (across the full range of intellectual ability) from birth until their 19th birthday. It covers the different ways that health and social care professionals can provide support, treatment, and help for children and young people with autism and their families and carers, from the early years through to their transition into young adult life.

Autism spectrum disorder in adults: diagnosis and management ${ }^{15}$

$$
\begin{aligned}
& \text { Challenging behaviour } \\
& \text { and learning } \\
& \text { disabilities: prevention } \\
& \text { and interventions for } \\
& \text { people with learning } \\
& \text { disabilities whose } \\
& \text { behaviour challenges }{ }^{16} \\
& \text { Mental health problems } \\
& \text { in people with learning } \\
& \text { disabilities: prevention, } \\
& \text { assessment and } \\
& \text { management }
\end{aligned}
$$

2012 This guideline covers diagnosing and managing suspected or confirmed autism spectrum disorder (autism, Asperger syndrome, atypical autism) in people 18 years and older. It aims to improve access and engagement with interventions and services as well as the experience of care for people with autism.

2015 This guideline covers interventions and support for children, young people, and adults with a learning disability and behavior that challenges. It highlights the importance of understanding the cause of behavior that challenges and performing thorough assessments so that steps can be taken to help people change their behavior and improve their quality of life. The guideline also covers support and intervention for family members or carers.

2016 This guideline covers preventing, assessing, and managing mental health problems in people with learning disabilities in all settings (including health, social care, education, and forensic and criminal justice). It aims to improve assessment and support for mental health conditions and help people with learning disabilities and their families and carers to be involved in their care.

Abbreviations: CG, Clinical Guideline; KCE, Belgian Healthcare Knowledge Centre; NG, NICE Guideline; NICE, National Institute for Health and Care Excellence; SIGN, Scottish Intercollegiate Guidelines Network.

guidelines, there were 524 recommendations in total, 270 of which related to intervention, with only 83 based on empirical evidence. Although intervention recommendations are the focus of this report, it is noteworthy that $45 \%$ of ASD and $58 \%$ of ID recommendations were not categorized as intervention (ie, assessment, general care, residential care, care related to transition periods). Within the category of intervention, most recommendations primarily comprised biomedical, pharmacologic, and psychosocial interventions for ASD and behavioral, pharmacologic, and psychological interventions for ID, with pharmacologic as the largest category for both conditions.

With respect to the quality of the recommendations, the level of detail varied across recommendations and guidelines. Recommendations were generally classifiable in relation to the type of provider trained to carry them out (eg, 


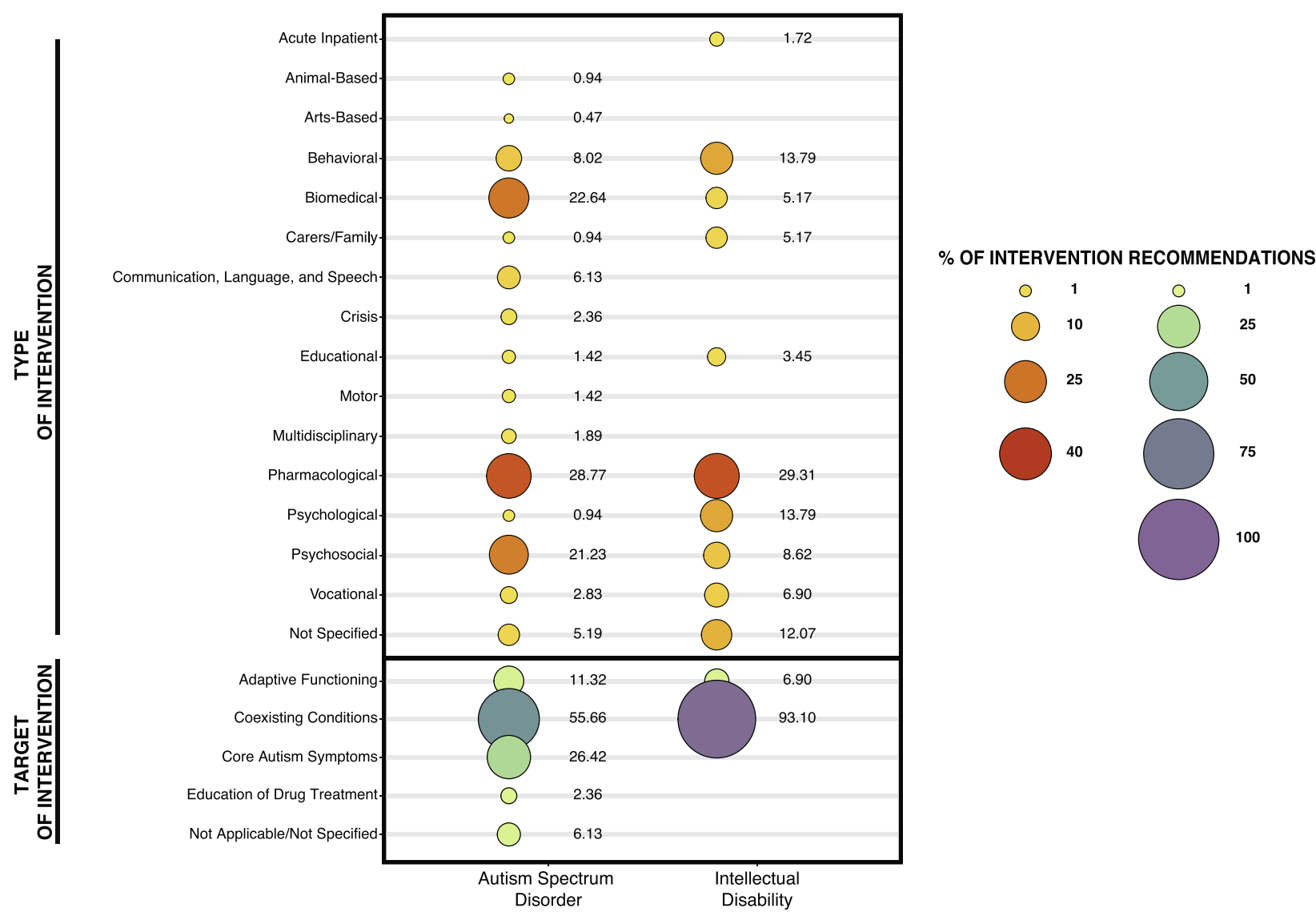

DIAGNOSTIC STATUS

Fig 2 Percentage of intervention recommendations organized by Type of Intervention and Target of Intervention for ASD and ID. Recommendations could be classified into more than 1 category.

medical provider, mental health provider, behavior therapist) but had limited information regarding skill level or training necessary to feasibly implement the recommended interventions. Additionally, the specificity of the recommendations in terms of the context or particular subpopulations for which they could be applicable was insufficient. Details regarding dosage, intensity, and duration of recommendations were usually not provided. In addition, few recommendations mentioned specific measures or programs; rather, most used general intervention categories (eg, classroombased interventions, interventions to support communication, sleep interventions). Finally, and perhaps most important to highlight, the majority of recommendations were based on expert opinion rather than empirical evidence (eg, randomized controlled trials).

Regarding the intervention recommendations that were based on empirical evidence, some generalizations can be made for each diagnostic group. For individuals with ASD, a vast array of medical and behavioral interventions have been developed and tested for different treatment targets and subpopulations, with varying degrees of rigor and efficacy. ${ }^{18,19}$ Specifically, from these guidelines, it was recommended to address (1) challenging behavior with antipsychotic medication only after other interventions have been unsuccessful; (2) mental health concerns with selective serotonin reuptake inhibitors or cognitive behavioral therapy (group or individual); (3) social communication and interaction problems with social learning programs (group or individual); (4) social isolation with structured leisure activity programs (group or individual); (5) employment needs with individually supported employment programs; (6) sleep difficulties with melatonin only after unsuccessful behavioral interventions; and (7) increased independence, communication, and social skills with applied behavioral analysis techniques.

For individuals with ID, recommendations on management often included general (nonindividualized) principles. ${ }^{20}$ From the guidelines reported here, intervention recommendations based on empirical evidence were in reference to (1) challenging behavior; (2) mental health problems; and (3) sleep problems. For challenging behavior, it was generally stated that individuals with ID benefit from structured daily routines, cognitive behavioral therapy (specifically for anger management and depression), parent training groups, exposure and relaxation therapy (for anxiety and phobias), and antipsychotic medication (only if other behavioral interventions have not been effective or if it is a high-risk problem behavior). Regarding the use of psychotropic medications, it was recommended that specialists, such as psychiatrists or developmental and behavioral 


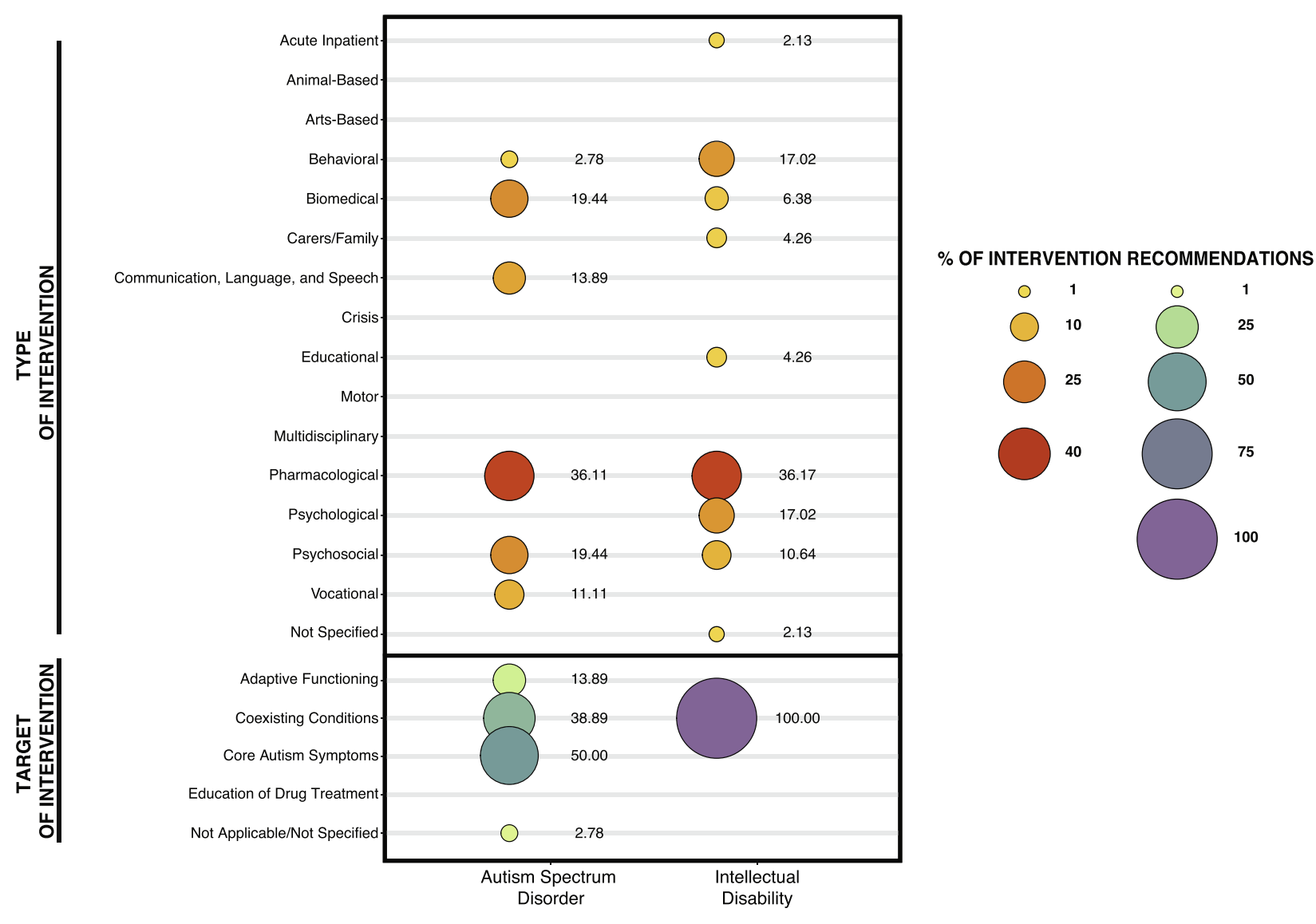

DIAGNOSTIC STATUS

Fig 3 Percentage of intervention recommendations based on empirical evidence organized by Type of Intervention and Target of Intervention for ASD and ID. Recommendations could be classified into more than 1 category.

pediatricians, prescribe these medications. It was also recommended that proactive strategies be used as much as possible to limit the use of reactive strategies. In the rare case that restrictive interventions must be used, they should be conducted ethically, documented, and reviewed regularly to determine whether continued use is warranted. For children aged 3-5 years who are at risk for or already have emerging challenging behavior, classroom-based interventions were recommended. Lastly, for sleep problems, behavioral interventions were recommended as the first approach and then medications as the last resort (with melatonin a preferred choice).

Coexisting conditions were the main target of intervention recommendations for both ASD and ID. This is partly because the ID guidelines concentrated specifically on challenging behavior and mental health problems; however, it underscores the fact that there exist few intervention guidelines targeting the core symptoms of ASD or ID. For both conditions, lack of sensitive outcome measures and rigorously controlled studies have impeded research efforts targeting core symptoms. ${ }^{21-23}$ One exception to this may be social skills because there were a number of interventions targeting these deficits (eg, speech/language/communication) in the guidelines. However, very few intervention recommendations were proffered for the other core symptoms of ASD (eg, restricted and repetitive patterns of behavior) or ID (low intellectual and adaptive functioning). This is a noteworthy gap because interventions for core symptoms could improve individuals' quality of life and ability to participate in the community.

In general, the lack of focus on adaptive behavior as an intervention target was surprising. Further examination of data contributing to this should focus on whether studies targeting such skills failed or were not implemented in the first place. Because of the major challenge of symptom heterogeneity, an individually-tailored intervention approach is often used; recommendations based on empirical evidence are still being developed as the field continues to be enhanced through improved emerging approaches, treatment research designs, and outcome measures. ${ }^{24-27}$ This may be why so many of the recommendations are for specific approaches to a particular problem or point of care rather than for a holistic approach to intervention.

Although some guidelines focused on specific subpopulations (eg, children, adults) or on specific targets (eg, challenging behaviors, mental health problems), a critical finding of this review was the need to consider more refined subpopulations within (or across) ASD and ID. Specifically, because the service needs of any given individual are fluid and dynamic, one necessity is to provide recommendations 
that can be tailored according to individual needs. This involves contextualizing recommendations on age, severity of core symptoms, cognitive ability, language level, adaptive functioning, support needs, comorbid symptom profile, sociocultural context, and/or known etiologies/syndromes. The significant heterogeneity in symptom presentation across ASD and ID and over time contributes to the diversity of rehabilitative needs, which negates the notion of a "one size fits all" guideline. Furthermore, functional deficits of either condition may indicate that recommendations developed for one disorder may actually be appropriate for the other, and it is a goal of the Package of Interventions for Rehabilitation to have recommendations that are designed to cut across various conditions and be grouped by functioning domains. ${ }^{1}$ This is particularly relevant for ASD and ID given their likelihood of co-occurrence, the prevalence of similar comorbidities, and patterns of adaptive functioning deficits.

Although the term "rehabilitation" suggests that a loss of functioning or a significant deviation from baseline occurred, which is the case in most conditions selected for the Package of Interventions for Rehabilitation (eg, spinal cord injury, stroke, traumatic brain injury), the WHO defines rehabilitation as a set of interventions designed to optimize functioning and reduce disability in individuals with health conditions in interaction with their environment. ${ }^{3}$ Rehabilitation can begin as soon as a condition is identified, including in early childhood. Thus, terms typically used in the developmental disability field such as "habilitation" and "treatment" can be interpreted as rehabilitation from a WHO perspective because their goal is to optimize functioning.

Services traditionally conceptualized as rehabilitative, including speech therapy, occupational therapy, and physical therapy, have long been among the most frequently used in targeting function in both ASD and ID. ${ }^{28}$ Moreover, the broader definition of rehabilitation used by the WHO allows for other types of interventions (eg, behavioral therapy, psychopharmacologic interventions). Neither condition has Food and Drug Administration or otherwise approved treatments for improving core symptoms, and traditionally considered rehabilitative services have also not proven to be efficacious in improving core symptoms. ${ }^{29}$ Therefore, the current ASD and ID guidelines presented here have mostly focused on providing general recommendations for treating conditions and behavior that frequently co-occur with these disorders but are not universal or specific to either diagnosis.

The next step in the larger WHO initiative involves development groups composed of members from all WHO regions for each respective condition to (1) select interventions from those identified from the clinical practice guidelines to be included in the Package of Interventions for Rehabilitation; (2) identify essential interventions that are missing in the selected guidelines; and (3) describe required resources (workforce, assistive technologies, equipment, consumables) and assignment to service delivery platforms for the interventions that are selected. For the selection of the interventions, the specific focus involves taking into account the available evidence, the number of people who will benefit from having access to interventions, and a good cost-benefit ratio. This process will include consideration of the inherent structural issues related to extrapolating guidelines from high-income countries to low- and middle-income countries when working toward a global health movement.

\section{Study limitations}

Although we consider this an extensive review of the guideline literature, there are still some limitations. It is possible that we may have had an incomplete retrieval of clinical practice guidelines that contain relevant intervention recommendations in ASD and ID because of the selection of search databases and restrictions on publication year (guidelines published in the last 10 years) and language (Englishonly guidelines). Although guidelines were only included in this review if they were written in (or already translated to) English, guidelines could be developed from studies published in any language. Because the goal of the Package of Interventions for Rehabilitation is to apply the recommendations from these guidelines globally, the WHO Rehabilitation Programme has composed a development group of members from all WHO regions, including those from low- and middleincome contexts, for the selection of intervention recommendations in an attempt to mitigate the effects of this English-only restriction. However, it is important to emphasize that recommendations extracted from these guidelines published in English may not be representative of all countries, contexts, or demographics. Another limitation is that we made inferences about the types and targets of service contained within each recommendation because this information was not always explicitly stated in the guidelines. It is also possible that interventions for ID may not have been included in guidelines because they lack information based on parsed specific genetic conditions that are associated with ID. ${ }^{30}$ Lastly, our decision to combine recommendations supported by each level of empirical evidence into 1 group (to compare findings across guidelines) limits the ability to delineate recommendations based on higher-level evidence from those based on lower-level evidence.

\section{Conclusions}

In sum, the diagnoses of ASD and ID alone do not directly translate into a common set of treatment targets. The Package of Interventions for Rehabilitation will include the sets of interventions targeting aspects of functioning that are relevant to people with the different diagnoses. This begs the question of the utility of guidelines based on diagnosis alone vs recommendations that can be tailored according to the behavioral needs of each individual. We argue that guidelines related to the provision of interventions for rehabilitation should be organized and implemented based on behavior or functioning rather than diagnosis.

\section{Corresponding author}

Audrey Thurm, PhD, Magnuson Clinical Center, Rm 1C250, MSC 1255, Bethesda, MD 20814. E-mail address: athurm@mail.nih.gov. 


\section{References}

1. Rauch A, Negrini S, Cieza A. Toward strengthening rehabilitation in health systems: methods used to develop a WHO package of rehabilitation interventions. Arch Phys Med Rehab 2019;100:2205-11.

2. Cieza A. Rehabilitation the health strategy of the 21 st century, really? Arch Phys Med Rehabil 2019;100:2212-4.

3. World Health Organization. Rehabilitation 2030 - a call for action. Available at: https://www.who.int/disabilities/care/Rehab2030MeetingReport2. pdf?ua=1. Accessed January 22, 2021.

4. Vos T, Abajobir AA, Abate $\mathrm{KH}$, et al. Global, regional, and national incidence, prevalence, and years lived with disability for 328 diseases and injuries for 195 countries, 1990-2016: a systematic analysis for the global burden of disease study 2016. Lancet 2017;390:1211-59.

5. American Psychiatric Association. Diagnostic and statistical manual of mental disorders. 5th ed. Arlington: American Psychiatric Association; 2013.

6. World Health Organization. International classification of diseases for mortality and morbidity statistics (11th revision). Available at: https://icd.who.int/browse11/l-m/en. Accessed January 22, 2021.

7. Maenner MJ, Shaw KA, Baio J, et al. Prevalence of autism spectrum disorder among children aged 8 years - Autism and Developmental Disabilities Monitoring Network, 11 sites, United States, 2016. MMWR Surveill Summ 2020;69:1-12.

8. Lai MC, Lombardo MV, Baron-Cohen S. Autism. Lancet 2014;383:896-910.

9. Lord C, Elsabbagh M, Baird G, Veenstra-Vanderweele J. Autism spectrum disorder. Lancet 2018;392:508-20.

10. Cooper SA, McLean G, Guthrie B, et al. Multiple physical and mental health comorbidity in adults with intellectual disabilities: population-based cross-sectional analysis. BMC Fam Pract 2015; $16: 110$

11. Brouwers MC, Kho ME, Browman GP, et al. AGREE II: advancing guideline development, reporting and evaluation in health care. CMAJ 2010;182:E839-42.

12. Scottish Intercollegiate Guidelines Network (SIGN). Assessment, diagnosis and interventions for autism spectrum disorders: a national clinical guideline. SIGN publication no. 145. Available at: http: / / www.sign.ac.uk. Accessed June 20, 2019.

13. Veereman G, Holdt Henningsen K, Eyssen M, et al. Management of autism in children and young people: a good clinical practice guideline. KCE reports 233. Available at: https://kce.fgov.be/ report/233. Accessed June 20, 2019.

14. National Institute for Health and Care Excellence (NICE). Autism spectrum disorder in under 19s: support and management. Clinical guideline CG170. Available at: https://www.nice.org.uk/ guidance/cg170. Accessed June 20, 2019.

15. National Institute for Health and Care Excellence (NICE). Autism spectrum disorder in adults: diagnosis and management. Clinical guideline CG142. Available at: https://www.nice.org.uk/ guidance/cg142. Accessed June 20, 2019.
16. National Institute for Health and Care Excellence (NICE). Challenging behaviour and learning disabilities: prevention and interventions for people with learning disabilities whose behaviour challenges. NICE guideline NG11. Available at: https:// www.nice.org.uk/guidance/ng11. Accessed June 20, 2019.

17. National Institute for Health and Care Excellence (NICE). Mental health problems in people with learning disabilities: prevention, assessment and management. NICE guideline NG54. Available at: https://www.nice.org.uk/guidance/ng54. Accessed June 20, 2019.

18. Williamson E, Sathe NA, Andrews JC, et al. AHRQ comparative effectiveness reviews. Medical therapies for children with autism spectrum disorder-an update. Rockville: Agency for Healthcare Research and Quality (US); 2017.

19. Weitlauf AS, McPheeters ML, Peters B, et al. Therapies for children with autism spectrum disorder: behavioral interventions update. 2014.

20. Patel DR, Cabral MD, Ho A, Merrick J. A clinical primer on intellectual disability. Transl Pediatr 2020;9(Suppl 1):S23-35.

21. Farmer C, Thurm A, Grant P. Pharmacotherapy for the core symptoms in autistic disorder: current status of the research. Drugs 2013;73:303-14.

22. Thurm A, Kelleher B, Wheeler A. Outcome measures for core symptoms of intellectual disability: state of the field. Am J Intellect Dev Disabil 2020;125:418-33.

23. Anagnostou $E$, Jones $N$, Huerta $M$, et al. Measuring social communication behaviors as a treatment endpoint in individuals with autism spectrum disorder. Autism 2015;19:622-36.

24. Baribeau DA, Anagnostou E. Social communication is an emerging target for pharmacotherapy in autism spectrum disorder - a review of the literature on potential agents. J Can Acad Child Adolesc Psychiatry 2014;23:20-30.

25. Lord C, Wagner A, Rogers S, et al. Challenges in evaluating psychosocial interventions for autistic spectrum disorders. J Autism Dev Disord 2005;35:695-708. [discussion 709-611].

26. National Autism Center. Findings and conclusions: National Standards Project, phase 2. Available at: https://www. nationalautismcenter.org/national-standards-project/phase2/. Accessed January 22, 2021.

27. Oliver PC, Piachaud J, Done J, Regan A, Cooray S, Tyrer P. Difficulties in conducting a randomized controlled trial of health service interventions in intellectual disability: implications for evidence-based practice. J Intellect Disabil Res 2002;46:340-5.

28. Wang L, Mandell DS, Lawer L, Cidav Z, Leslie DL. Healthcare service use and costs for autism spectrum disorder: a comparison between medicaid and private insurance. J Autism Dev Disord 2013;43:1057-64.

29. Ameis SH, Kassee C, Corbett-Dick P, et al. Systematic review and guide to management of core and psychiatric symptoms in youth with autism. Acta Psychiatr Scand 2018;138:379-400.

30. Vissers LE, Gilissen C, Veltman JA. Genetic studies in intellectual disability and related disorders. Nat Rev Genet 2015;17:9. 